\title{
Organizing Large Scale Hacking Competitions
}

Nicholas Childers

Giovanni Vigna

Ludovico Cavedon

Manuel Egele Lorenzo Cavallaro

Bryce Boe

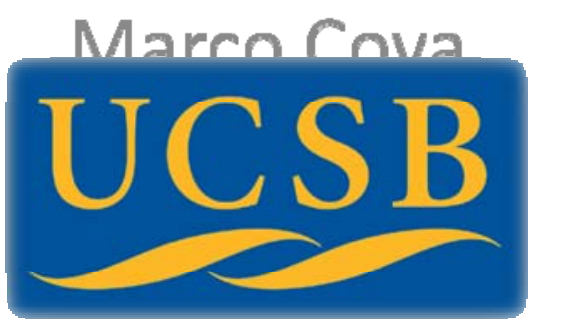




\section{Outline}

- Hacking Competitions Overview

- UCSB's iCTF

- History

- 2003-2007 Competitions

- 2008 Competition

- 2009 Competition

- Lessons Learned

- Final Remarks 


\section{HACKING COMPETITIONS OVERVIEW}




\section{Why a hacking competition?}

- Time constrained

- Provides hands-on security experience

- Mimics real-world scenarios

- It's fun

- Engaging

- Motivates students to go beyond the call of duty

- Promotes participation 


\section{Types of hacking competitions}

- Challenge based

- DEFCON Quals, Codegate

- Capture the flag

- DEFCON, iCTF 2003-2007, CIPHER, RUCTF 


\section{3-2007 iCTF example}

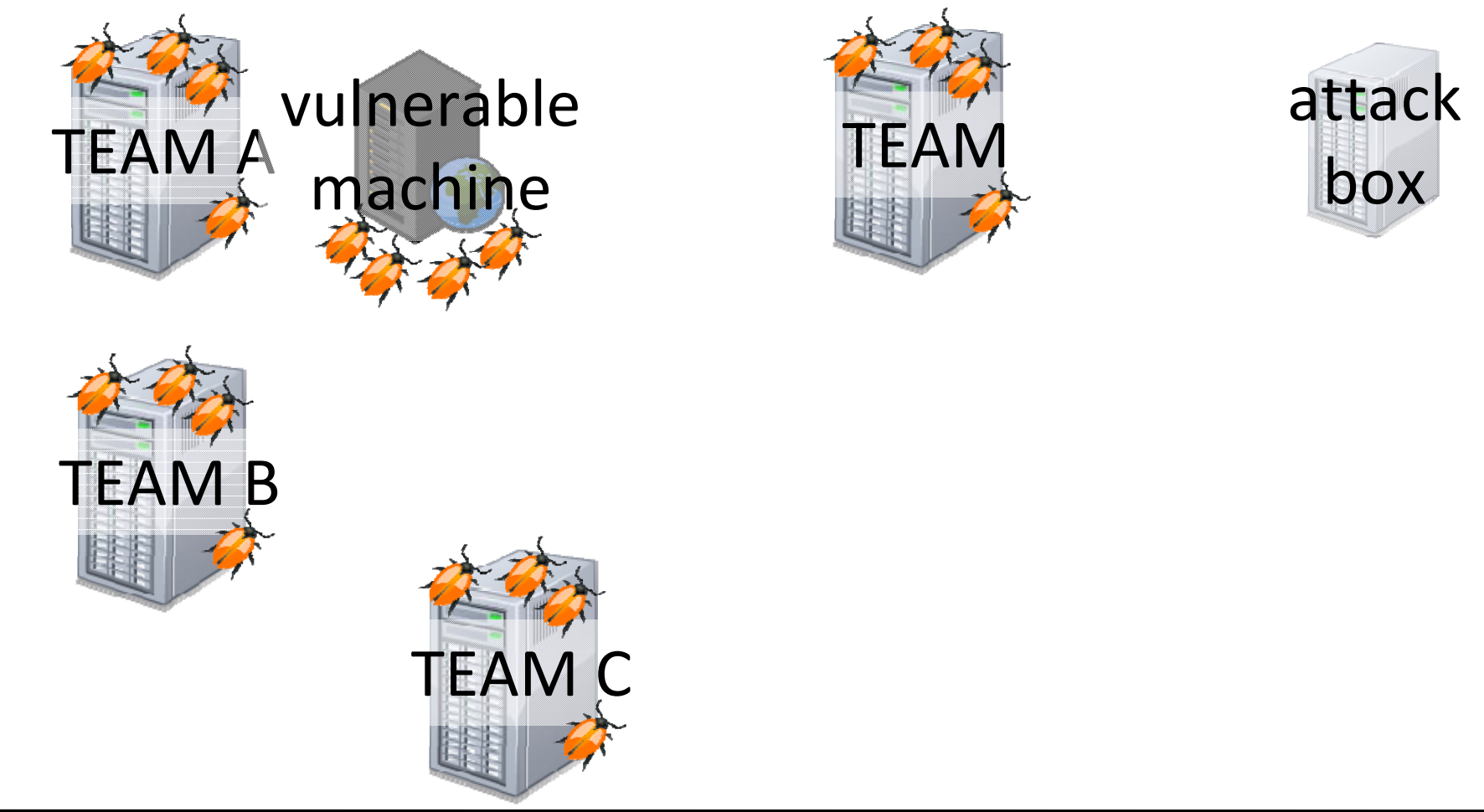




\section{3-2007 iCTF example}

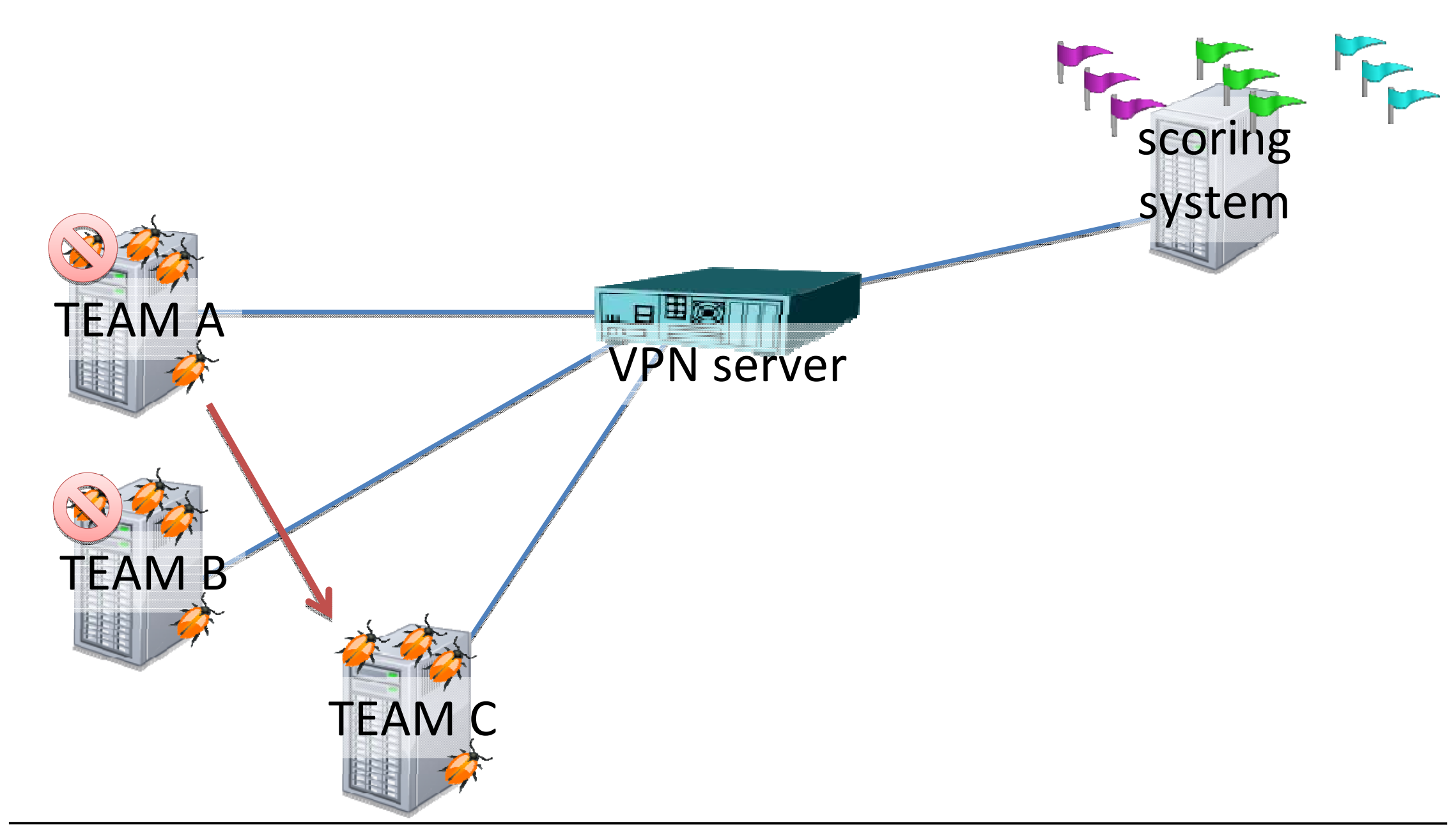




\section{Types of hacking competitions}

- Challenge based

- DEFCON Quals, Codegate

- Capture the flag

- DEFCON, iCTF 2003-2007, CIPHER, RUCTF

- Attack based

- Pwn2Own, iCTF 2008-2009

- Defense based

- Cyber Defense Exercise (CDX)

- NSF Security Grand Challenge 


\section{Hosting a hacking competition}

- Design

- Challenging but not frustrating

- Cater to various abilities

- Be objectively scored

- Development

- Allocate ample time

- Execution

- Maintain and monitor network

- Support remote teams

- Limited timeframe 


\section{UCSB'S INTERNATIONAL CAPTURE THE FLAG COMPETITION}




\section{iCTF History}

- 2003: 14 US university teams

- 2004: Addition of European teams

- 2005: Addition of more international teams

- 2006: 25 teams

- 2007: 36 teams

- 2008: 39 teams

- 2009: 56 teams 


\section{3-2007 Competitions}

- Traditional CTF format with side challenges

- Limited to universities

- Addition of remote teams

- Introduced traffic blending technique

Limitations

- Favored experienced teams

- No longer unique 


\section{8 iCTF}

- Attack-based with side challenges

- Mimics a "save the world" scenario

- Goal: Defuse bomb by breaking into the softerror.com network 


\section{Simulated Network}

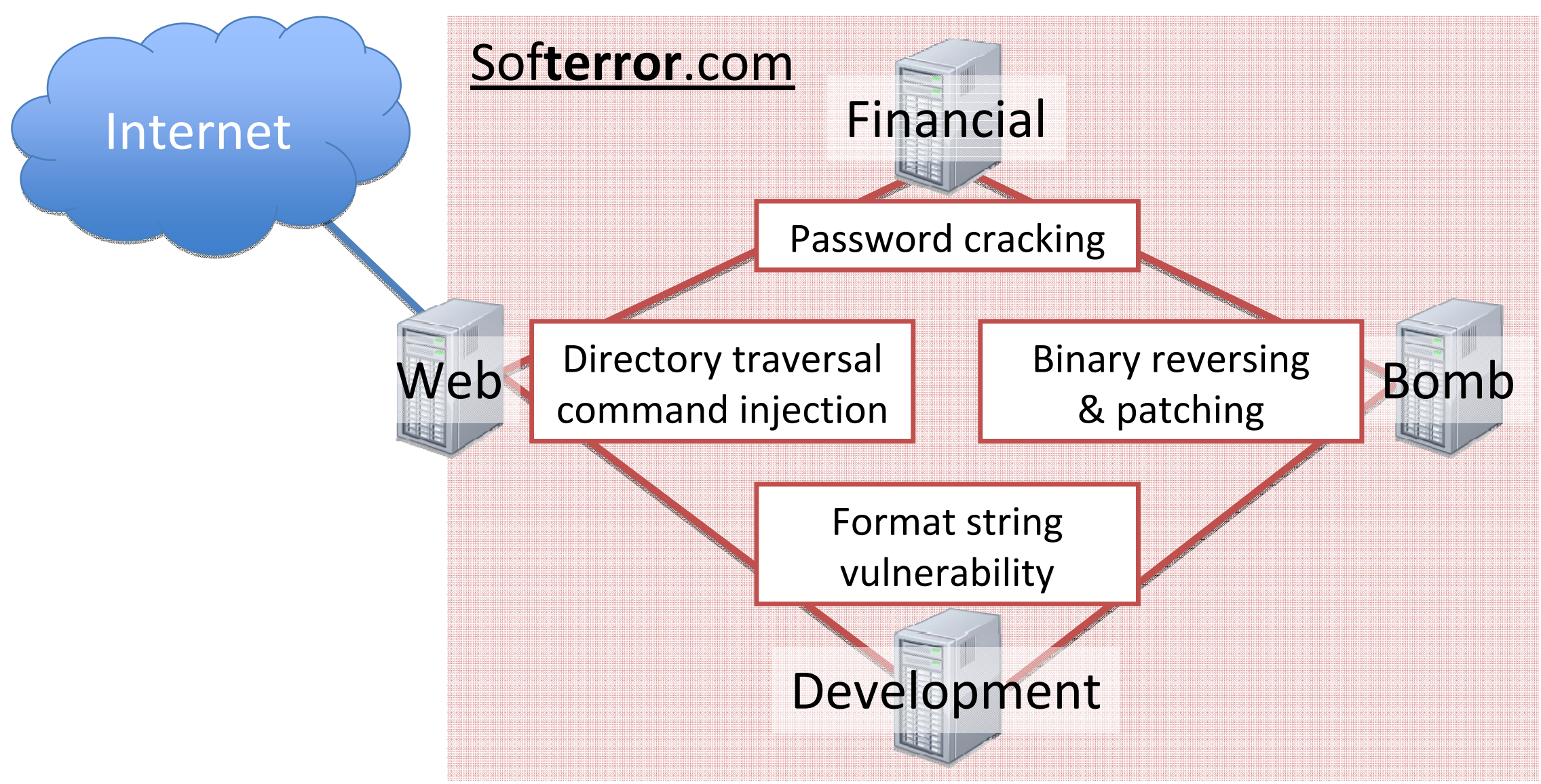




\section{Physical Network}

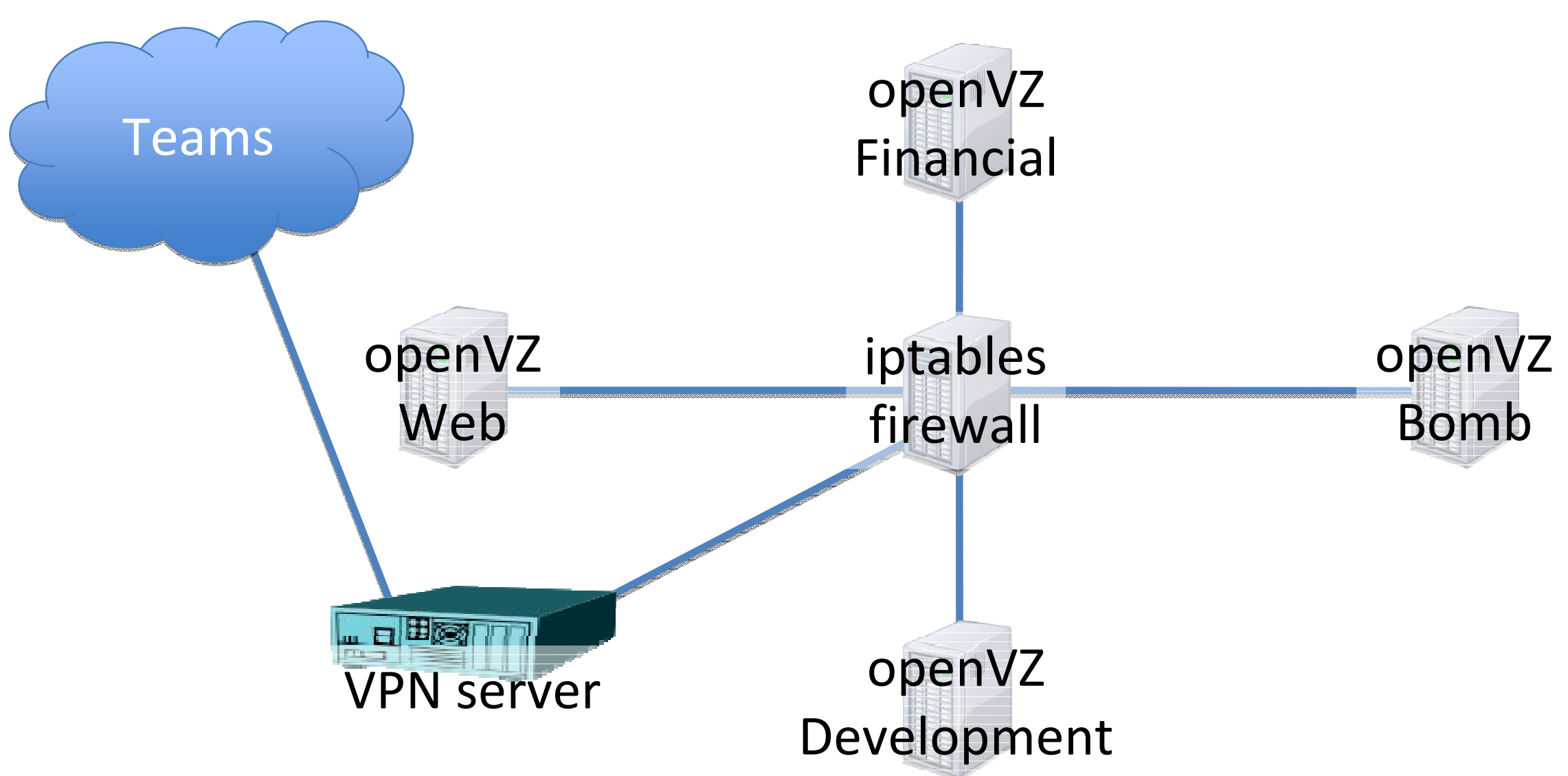




\section{Dataset}

- Snort Alerts (by team)

- Mean: 8482

- Max: 43254

- Pcap files

- 5.5 GB data (3 GB compressed)

- 34 million packets

- Useful for attack correlation research 


\section{9 iCTF}

- Also attack based with side challenges

- Mimics a "botnet creation" scenario

- Goal: Deliver profitable drive-by-downloads to simulated web users 


\section{Game Play}




\section{Game Play}

\section{UC Santa Barbara}

Goollable

PayPerNews

periodic indexing

optional publishing $\$ \$ \$$

Team Website

4 


\section{Game Play}

\section{UC Santa Barbara}

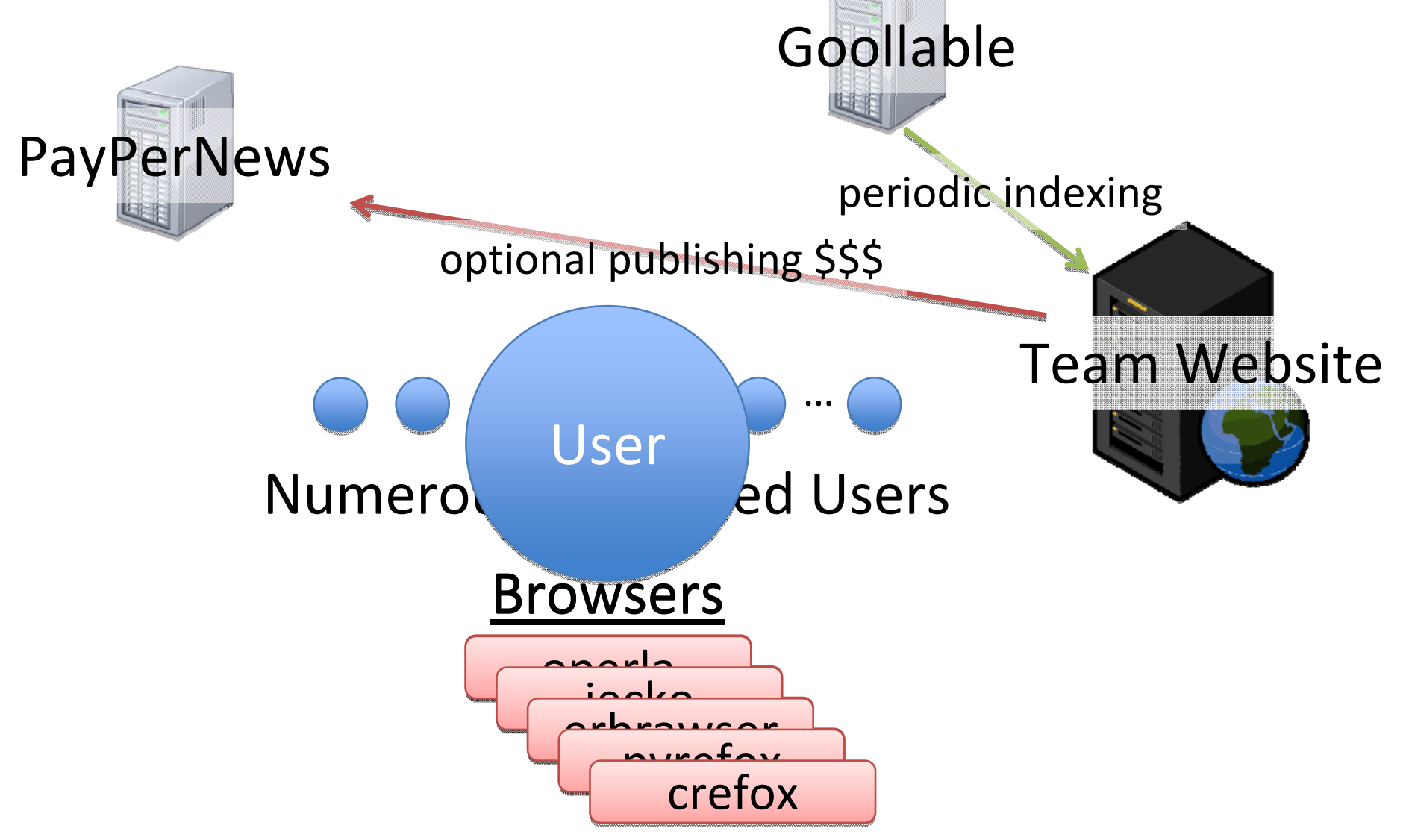




\section{Game Play}

\section{UC Santa Barbara}

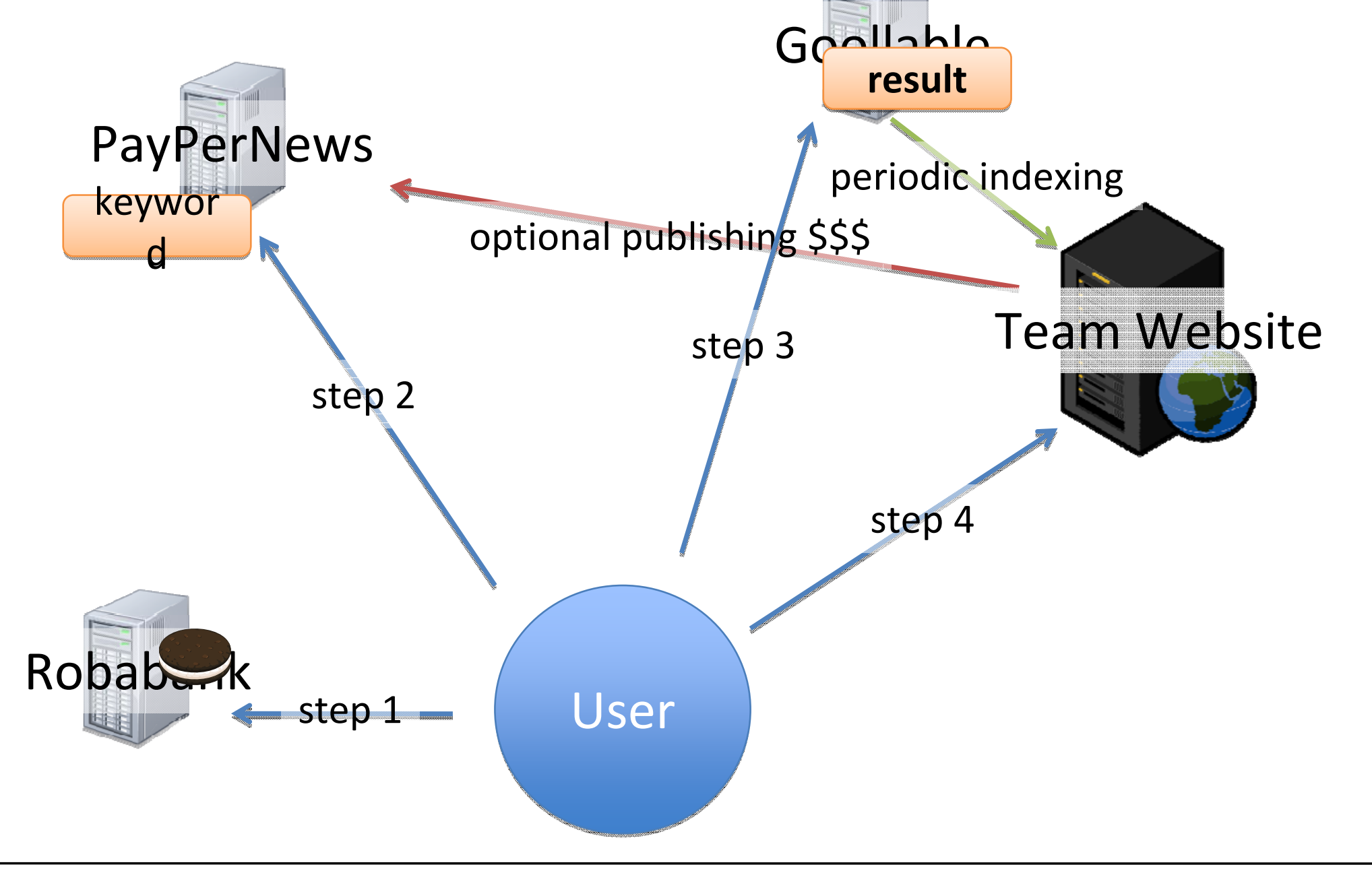

Bryce Boe @ UCSB -- DIMVA 2010 


\section{Game Play}

\section{UC Santa Barbara}

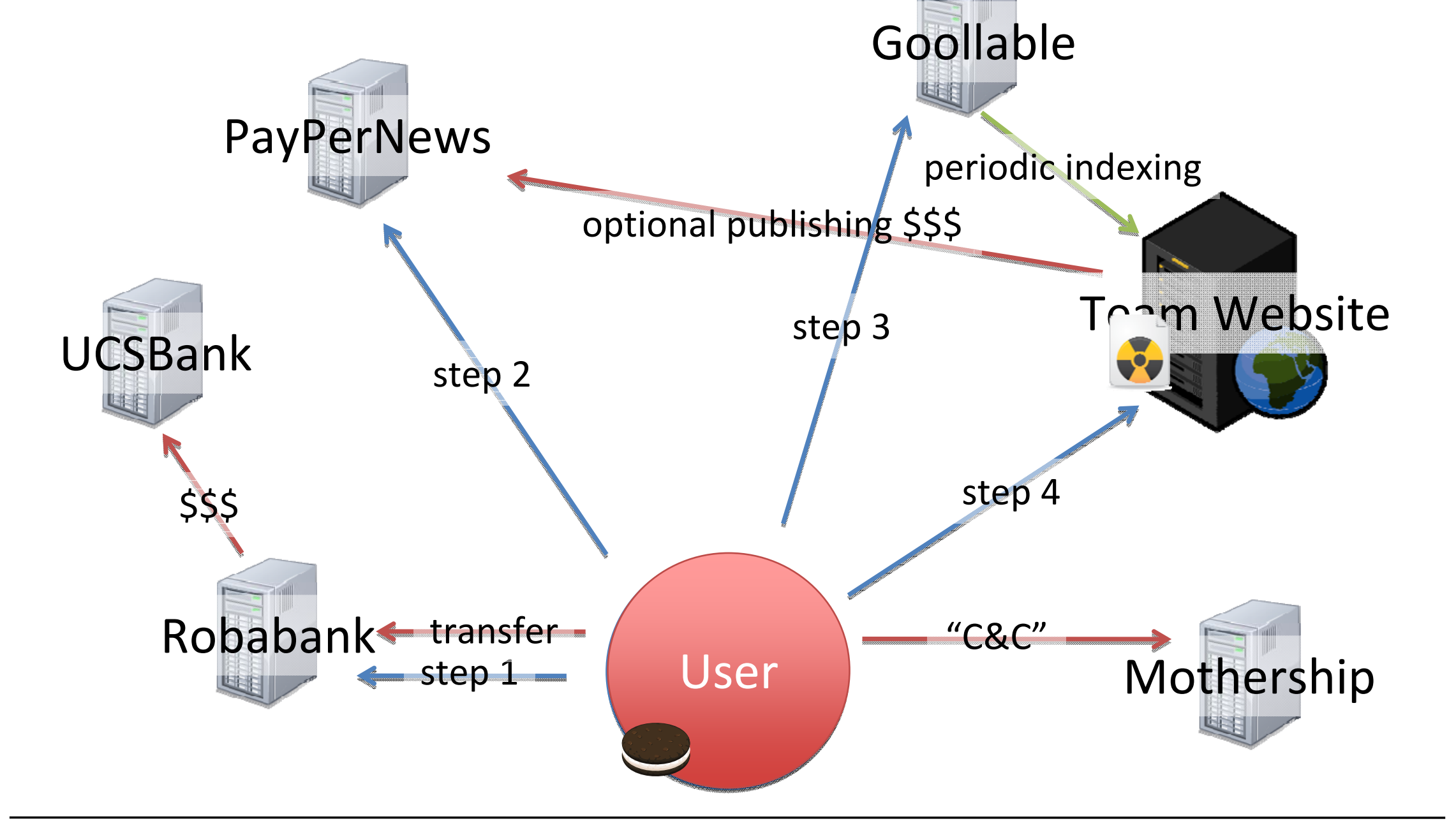




\section{Lessons Learned}

- KISS principle

- Budget sufficient time and resources

- Stress test competition components

- Scoring

- Fully automated

- Rollback and repeatable

- Attack only competitions level the playing field 


\section{Final Remarks}

- Hacking Competitions

- Fun and Challenging

- Engaging

- Datasets and source from UCSB's iCTF available at http://ictf.cs.ucsb.edu 


\section{Questions?}

UC Santa Barbara

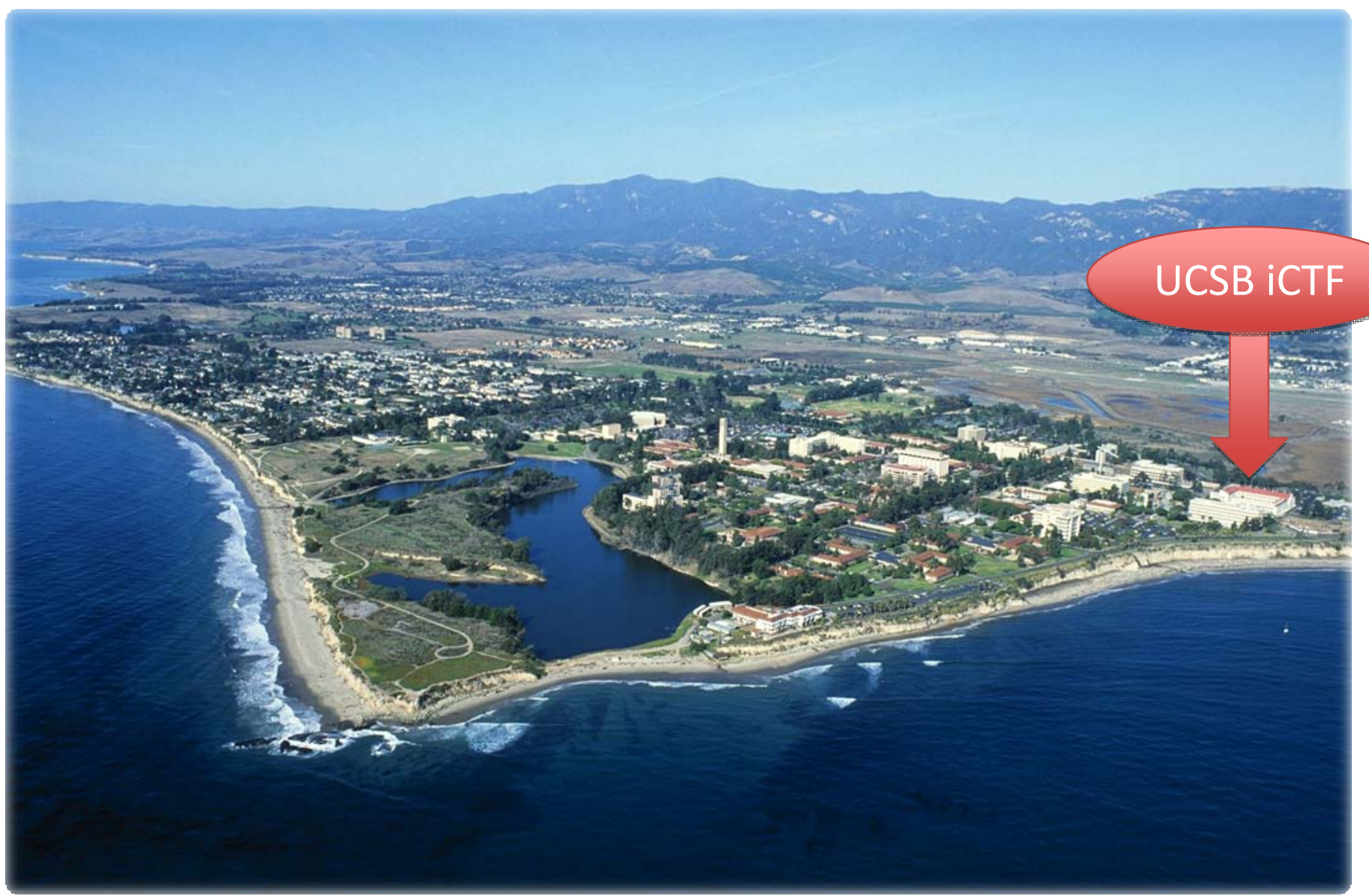

